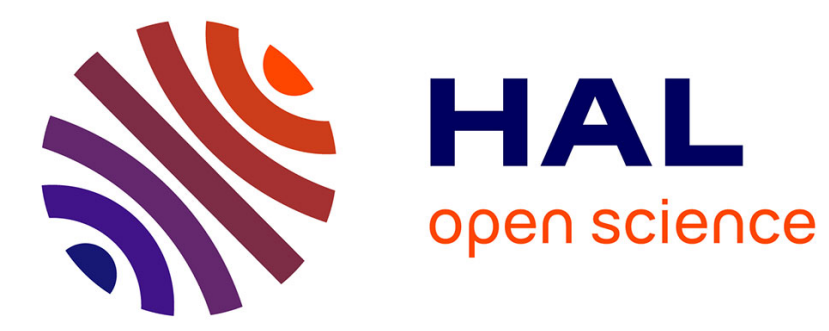

\title{
Public Spending as a Source of Endogenous Business Cycles in a Ramsey Model with Many Agents
} Kazuo Nishimura, Carine Nourry, Thomas Seegmuller, Alain Venditti

\section{To cite this version:}

Kazuo Nishimura, Carine Nourry, Thomas Seegmuller, Alain Venditti. Public Spending as a Source of Endogenous Business Cycles in a Ramsey Model with Many Agents. 2013. halshs-00796698

\section{HAL Id: halshs-00796698 \\ https://shs.hal.science/halshs-00796698}

Preprint submitted on 4 Mar 2013

HAL is a multi-disciplinary open access archive for the deposit and dissemination of scientific research documents, whether they are published or not. The documents may come from teaching and research institutions in France or abroad, or from public or private research centers.
L'archive ouverte pluridisciplinaire HAL, est destinée au dépôt et à la diffusion de documents scientifiques de niveau recherche, publiés ou non, émanant des établissements d'enseignement et de recherche français ou étrangers, des laboratoires publics ou privés. 




\section{Working Papers / Documents de travail}

Public Spending as a Source of Endogenous Business Cycles in a Ramsey Model with Many Agents

Kazuo Nishimura

Carine Nourry Thomas Seegmuller

Alain Venditti 


\title{
Public spending as a source of endogenous business cycles in a Ramsey model with many agents ${ }^{1}$
}

\author{
Kazuo NISHIMURA ${ }^{2}$, Carine NOURRY ${ }^{3,4}$, Thomas SEEGMULLER ${ }^{2}$ \\ and Alain VENDITTI 2,5
}

February 8, 2013

\footnotetext{
${ }^{1}$ We would like to thank the Associate Editor A. Serletis and two anonymous referees for useful comments and suggestions.

${ }^{2}$ Institute of Economic Research, Kyoto University

${ }^{3}$ Aix-Marseille University (Aix-Marseille School of Economics), CNRS-GREQAM, EHESS

${ }^{4} \mathrm{IUF}$

${ }^{5}$ EDHEC
} 


\begin{abstract}
We introduce public spending, financed through income taxation, in the Ramsey model with heterogeneous agents. Public spending as a source of welfare generates more complex dynamics. In contrast to previous contributions focusing on similar models but with wasteful public spending, limit cycles through Hopf bifurcation and expectation-driven fluctuations appear if the degree of capital-labor substitution is large enough to be compatible with capital income monotonicity. Moreover, unlike frameworks with a representative agent, our results do not require externalities in production and are compatible with a weakly elastic labor supply with respect to wage.

JEL classification: C62, E32, H23.
\end{abstract}

Keywords: Endogenous cycles, indeterminacy, heterogeneous agents, public spending, endogenous labor supply, borrowing constraint. 


\section{Introduction}

The Ramsey (1928) model is one of the most popular frameworks to analyze macroeconomic dynamics. As it is well-known, when there is one representative agent, one sector and usual assumptions (constant returns to scale, concave utility function, constant discount factor), the economy monotonically converges to the steady state.

With many agents, conclusions may differ. Introducing borrowing constraints, Becker (1980) shows the so-called Ramsey conjecture, i.e. the most patient agent holds the whole capital stock in the long run. Most importantly for our purpose, dynamics can be non-monotonic and endogenous cycles can occur around this steady state. As shown by Becker and Foias $(1987,1994)$, this requires the non-monotonicity of capital income: the capital income has to decrease with respect to capital, i.e. the inputs are sufficiently low substitutes. When labor supply is elastic, this conclusion can be slightly relaxed (Bosi and Seegmuller (2010a)), but endogenous cycles still require a sufficiently weak elasticity of capital-labor substitution. In these contributions, cycles have period two and expectation-driven fluctuations cannot occur.

Introducing public spending financed through a balanced-budget rule in the Ramsey model with many agents, indeterminacy, and therefore sunspot fluctuations can arise. This has been investigated by Bosi and Seegmuller (2010b), considering wasteful government expenditures and progressive taxation on capital and labor incomes. ${ }^{1}$ Their paper emphasises that the multiplicity of equilibria is mainly explained by the intertemporal choice of the most patient agent when affected by non-linear capital taxation. However,

\footnotetext{
${ }^{1}$ A similar result is obtained by Sorger (2002) in a model with progressive taxation, but without endogenous labor.
} 
endogenous fluctuations still require a low elasticity of capital-labor substitution, meaning again the non-monotonicity of capital income, which can be criticized from an empirical point of view, ${ }^{2}$ and still occur through a flip bifurcation and period-two cycles. It is worth pointing out that, under certain circumstances, this is likely to provide unsatisfactory empirical properties, such that negative auto-correlations of the main variables. This is typically the case, for instance, when the flip bifurcation is subcritical and the model is evaluated while a 2-period cycle surrounds the steady-state.

In this paper we still focus on government intervention as a source of expectationdriven fluctuations. But contrary to Bosi and Seegmuller (2010b), we consider that public spending is useful as it improves households' utility of consumption as an externality. In such a framework, we will show that even with linear taxation, the intertemporal choices of the most patient household are affected in such a way that sunspot fluctuations become compatible with capital income monotonicity, meaning plausible values of the elasticity of capital-labor substitution. The public spending externality is the main ingredient explaining endogenous business cycles. ${ }^{3}$ Indeed, following an optimistic expectation, the

\footnotetext{
${ }^{2}$ To fix ideas, non-monotonicity of capital income is obtained when the elasticity of capital-labor substitution $\sigma$ is lower than the expression $(1-s)\left[1-\beta_{1}(1-\delta)\right]$, with $s$ the share of capital in total income, $\beta_{1}$ the discount factor of the most patient agent and $\delta$ the depreciation rate of capital. Considering that at quarterly frequencies $s=0.3, \beta_{1}=0.99$ and $\delta=0.025$, we get $\sigma<0.0243$. Such a restriction is not compatible with all the recent estimates of the elasticity of substitution. For instance, Duffy and Papageorgiou [10] report robust estimates that are contained in $[1.24,3.24]$. On the contrary, LeónLedesma et al. $[18]$ provide robust estimates in the range $[0.4,0.6]$.

${ }^{3}$ Lloyd-Braga et al. (2008) also show that public spending can be a source of endogenous fluctuations when there are heterogeneous households. The Woodford (1986) framework considered is however different, since the results apply to a monetary equilibrium with an infinitely elastic labor supply with respect
} 
increase of investment generates two effects: on the one hand, the implied increase of the marginal rate of intertemporal substitution in consumption is mitigated by the presence of public spending externalities. On the other hand, these externalities also generate a more than porportional increase of the labor supply with respect to capital, implying a decrease of the capital-labor ratio and thus a higher interest rate. These two effects are then compatible with the Euler equation and expectations are self-fulfilling.

It is also worthwhile to note that endogenous deterministic fluctuations now emerge not only through a flip bifurcation (Guo and Harrison (2008)) but also through a Hopf bifurcation. As emphasized in Dufourt et al. [12], the two-dimensional dynamical system that describes the intertemporal equilibrium is then much more likely to provide a satisfactory account of observed business cycles (characterized by persistent fluctuations, positive auto-correlations and, usually, non-monotonic dynamics of convergence to the steady-state) since, close to a Hopf bifurcation, the eigenvalues of the Jacobian matrix evaluated at the steady-state are complex conjugates with a modulus close to one.

In this framework, we also emphasize the crucial role played by the endogenous labor supply which has to be elastic with respect to public spending, ${ }^{4}$ but can be weakly elastic with respect to the wage rate. This last property appears to be in accordance with the empirical evidence. ${ }^{5}$ We note that this is in contrast to models with public spending exto the wage.

${ }^{4}$ Note that in our framework, public spending externalities can be seen as a distortion on the labor market. See also Dufourt et al. (2008) and Grandmont (2008) where distortions on the labor market, such that unemployment benefits, unions or efficiency wages, also generate local indeterminacy under plausible values of parameters.

${ }^{5}$ At the microeconomic level, most econometric analysis available in the literature conclude that the 
ternalities and a representative consumer where an infinitely elastic labor supply is often assumed (see for instance Guo and Harrison (2008)). Another important difference with respect to the previous literature is that in our framework with heterogeneous households, deterministic cycles occur without requiring externalities and increasing returns in production (see for instance Zhang (2000)).

This paper is organized as follows. The model is presented in the next section. Section 3 is devoted to the steady state analysis. In Section 4 , we study the occurrence of endogenous business cycles. Section 5 provides some economic intuitions, while concluding remarks appear in Section 6. Proofs and technical details are provided in the Appendix.

\section{The model}

We consider a discrete time economy $(t=0,1, \ldots, \infty)$, with three types of agents, households, firms and a government.

\section{$2.1 \quad$ Households}

There are $H$ heterogeneous infinitely lived households, indexed by $i=1, \ldots, H$, who supply elastically labor and face borrowing constraints. They have heterogeneous capital endowments $\left(k_{i 0} \geqslant 0\right)$ and preferences, i.e. different discount factors and different

wage elasticity of labor belongs to $(0,0.5)$ for men and to $(0.5,1)$ for women (see Blundell and MaCurdy [6]). On the contrary, Rogerson and Wallenius [27] have shown that, due to the role of the participation decision of women and the extent of early retirement, the elasticities at the macroeconomic level are virtually unrelated to the micro elasticities. The macroeconomic elasticity of the labor supply with respect to the wage appears to be in the range of $(2.25,3.0)$. In both cases, the elasticity is not too large. 
instantaneous utilities in consumption and leisure. To fix ideas, households are ranked according to their discount factors: $0 \leq \beta_{H} \leq \ldots \leq \beta_{2}<\beta_{1}<1$.

Consumer $i$ has separable preferences over time, and between consumption and leisure. Moreover, we assume that public spending $G_{t}$ affects welfare, as an externality on utility for consumption. ${ }^{6}$ Denoting $c_{i t}$ his consumption and $l_{i t}$ his labor supply at period $t$, consumer i's utility function is given by:

$$
\sum_{t=0}^{+\infty} \beta_{i}^{t}\left[u_{i}\left(c_{i t}, G_{t}\right)-v_{i}\left(l_{i t}\right)\right]
$$

Denote $r_{t}$ the real interest rate, $w_{t}$ the real wage, $\delta \in(0,1)$ the depreciation rate of capital and $\tau \in(0,1)$ the constant tax rate on income. Each household maximizes (1) facing the budget constraint:

$$
c_{i t}+k_{i t+1}-(1-\delta) k_{i t}=(1-\tau)\left(r_{t} k_{i t}+w_{t} l_{i t}\right)
$$

the borrowing constraint on individual capital holding $k_{i t} \geq 0$ and the constraint on labor $l_{i t} \in[0, \bar{\ell}]$, with $\bar{\ell}$ the endowment of labor. The utility function satisfies the following assumption:

Assumption 1. $u_{i}\left(c_{i}, G\right)$ and $v_{i}\left(l_{i}\right)$ are continuous functions defined on $[0,+\infty) \times$ $[0,+\infty)$ and $[0, \bar{\ell}]$, and $C^{2}$ on $(0,+\infty) \times(0,+\infty)$ and $(0,1)$, respectively. $u_{i}\left(c_{i}, G\right)$ is strictly increasing $\left(u_{i c}\left(c_{i}, G\right)>0\right)$ and strictly concave $\left(u_{i c c}\left(c_{i}, G\right)<0\right)$ with respect to

\footnotetext{
${ }^{6}$ One could also introduce a public spending externality in the disutility of labor. Since we will focus on equilibria where the most patient household supplies no labor, it would not alter his behavior. This would only affect the relationship between labor supply and public spending coming from impatient households' behavior $(i=2, \ldots, H)$. This link would be reinforced if the marginal disutility of labor decreases with the public spending externality, and dampened otherwise.
} 
its first argument. ${ }^{7} v_{i}\left(l_{i}\right)$ is strictly increasing $\left(v_{i}^{\prime}\left(l_{i}\right)>0\right)$ and convex $\left(v_{i}^{\prime \prime}\left(l_{i}\right) \geqslant 0\right)$. In addition, the Inada conditions $\lim _{c_{i} \rightarrow 0} u_{i 1}\left(c_{i}, G\right)=+\infty, \lim _{l_{i} \rightarrow \bar{\ell}} v_{i}^{\prime}\left(l_{i}\right)=+\infty$ are satisfied.

Utility maximization gives:

$$
\begin{aligned}
& u_{i c}\left(c_{i t}, G_{t}\right)(1-\tau) w_{t} \leq v_{i}^{\prime}\left(l_{i t}\right), \text { with equality when } l_{i t}>0 \\
& \frac{u_{i c}\left(c_{i t}, G_{t}\right)}{u_{i c}\left(c_{i t+1}, G_{t+1}\right)} \geq \beta_{i} R_{t+1} \text {, with equality when } k_{i t+1}>0
\end{aligned}
$$

with $R_{t+1}=1-\delta+(1-\tau) r_{t+1}$ and the transversality condition $\lim _{t \rightarrow+\infty} \beta_{i}^{t} u_{i c}\left(c_{i t}, G_{t}\right) k_{i t+1}=0$. For all $i=1, \ldots, H, c_{i t}$ and $l_{i t}$ are forward variables while $k_{i t}$ are predetermined variables. For further reference, we introduce the following elasticities: ${ }^{8}$

$$
\epsilon_{i c c} \equiv-u_{i c c} c_{i} / u_{i c}>0, \epsilon_{i c G} \equiv u_{i c G} G / u_{i c}, \epsilon_{i l l} \equiv v_{i}^{\prime \prime} l_{i} / v_{i}^{\prime} \geqslant 0
$$

\subsection{Firms}

A representative firm produces the final good $y_{t}$, using a technology with constant returns $y_{t}=F\left(k_{t}, l_{t}\right)=f\left(a_{t}\right) l_{t}$, where $k_{t}$ denotes capital, $l_{t}$ labor and $a_{t} \equiv k_{t} / l_{t}$. The intensive production function $f(a)$ satisfies:

Assumption 2. $f(a)$ is a continuous function defined on $[0,+\infty)$ and $C^{2}$ on $(0,+\infty)$, strictly increasing $\left(f^{\prime}(a)>0\right)$ and strictly concave $\left(f^{\prime \prime}(a)<0\right)$. In addition, the conditions $\lim _{a \rightarrow 0} f^{\prime}(a)=+\infty$ and $\lim _{a \rightarrow+\infty} f^{\prime}(a)<\theta /\left(\beta_{1}(1-\tau)\right)$ are satisfied, where $\theta \equiv 1-\beta_{1}(1-\delta)$

Profit maximization gives:

$$
r_{t}=f^{\prime}\left(a_{t}\right) \equiv r\left(a_{t}\right) \quad \text { and } \quad w_{t}=f\left(a_{t}\right)-a_{t} f^{\prime}\left(a_{t}\right) \equiv w\left(a_{t}\right)
$$

Due to constant returns to scale and perfect competition, profits are zero, i.e.,

${ }^{7}$ We denote $u_{i x_{j}}\left(x_{1}, x_{2}\right)=\partial u_{i}\left(x_{1}, x_{2}\right) / \partial x_{j}$ and $u_{i x_{j} x_{h}}\left(x_{1}, x_{2}\right)=\partial^{2} u_{i}\left(x_{1}, x_{2}\right) / \partial x_{j} \partial x_{h}$.

${ }^{8}$ For simplicity, we omit the arguments of the functions. 


$$
f\left(a_{t}\right) l_{t}=w_{t} l_{t}+r_{t} k_{t}
$$

In the following, we denote by $s(a) \equiv a f^{\prime}(a) / f(a) \in(0,1)$ the capital share in total income and $\sigma(a) \equiv[s(a)-1] f^{\prime}(a) /\left[a f^{\prime \prime}(a)\right] \geq 0$ the elasticity of capital-labor substitution. We derive the following useful relationships:

$$
r^{\prime}(a) a / r(a) \equiv-(1-s(a)) / \sigma(a) \quad \text { and } \quad w^{\prime}(a) a / w(a) \equiv s(a) / \sigma(a)
$$

\subsection{Government}

Public spending $G_{t}$ is financed by income taxation, through a balanced-budget rule:

$$
G_{t}=\tau \sum_{i=1}^{H}\left(r_{t} k_{i t}+w_{t} l_{i t}\right)
$$

where $\tau \in(0,1)$ is the constant proportional tax rate on households' income.

\subsection{Intertemporal equilibrium}

An intertemporal equilibrium can be defined as follows:

Definition 1. Under Assumptions 1 and 2, an equilibrium of the economy $E=$ $\left(f, \tau,\left(k_{i 0}, \beta_{i}, u_{i}, v_{i}\right)_{i=1}^{H}\right)$ is a sequence $\left(r_{t}, w_{t}, k_{t}, l_{t}, G_{t},\left(k_{i t}, l_{i t}, c_{i t}\right)_{i=1}^{H}\right)_{t=0}^{+\infty}$ satisfying the following conditions:

(D1) $k_{t}>0, l_{t}>0, k_{i t} \geq 0,0 \leq l_{i t}<\bar{\ell}, c_{i t}>0{ }^{9}$

(D2) $r_{t}=f^{\prime}\left(a_{t}\right) \equiv r\left(a_{t}\right)$ and $w_{t}=f\left(a_{t}\right)-a_{t} f^{\prime}\left(a_{t}\right) \equiv w\left(a_{t}\right)$, with $a_{t}=k_{t} / l_{t}$;

(D3) $u_{i c}\left(c_{i t}, G_{t}\right)(1-\tau) w_{t} \leq v_{i}^{\prime}\left(l_{i t}\right)$, with equality when $l_{i t}>0$;

(D4) $u_{i c}\left(c_{i t}, G_{t}\right) \geq \beta_{i} R_{t+1} u_{i c}\left(c_{i t+1}, G_{t+1}\right)$, with equality when $k_{i t+1}>0$, and $R_{t+1} \equiv$ $R\left(a_{t+1}\right)=1-\delta+(1-\tau) r\left(a_{t+1}\right) ;$

(D5) $c_{i t}+k_{i t+1}-(1-\delta) k_{i t}=(1-\tau)\left(r_{t} k_{i t}+w_{t} l_{i t}\right)$;

\footnotetext{
${ }^{9}$ Assumption 1 rules out any equilibrium with $c_{i t}=0$ or $l_{i t}=\bar{\ell}$.
} 
(D6) $k_{t}=\sum_{i=1}^{H} k_{i t}$;

(D7) $l_{t}=\sum_{i=1}^{H} l_{i t}$

(D8) $G_{t}=\tau \sum_{i=1}^{H}\left(r_{t} k_{i t}+w_{t} l_{i t}\right)$;

and the transversality condition $\lim _{t \rightarrow+\infty} \beta_{i}^{t} u_{i c}\left(c_{i t}, G_{t}\right) k_{i t+1}=0$, for $i=1, \ldots H$.

The existence of the intertemporal equilibrium is an issue that we do not address in this paper. The interested reader can refer to Becker et al. (1991), Bosi and Seegmuller (2010a) or Becker et al. (2012). In the next section, we show the existence of a steady state. Since we focus on local dynamics around such an equilibrium, we consider that, by continuity, an intertemporal equilibrium exists in a neighborhood of the steady state.

\section{$3 \quad$ Steady state analysis}

Since the tax rate on income is constant, the most patient household holds all the capital stock at the steady state (Becker (1980)). Moreover, two types of steady states may exist, depending on whether the most patient household supplies labor or not (Bosi and Seegmuller (2010a)).

Proposition 1. Under Assumptions 1 and 2, there exists a steady state defined by the following properties:

(S1) $r=f^{\prime}(a), w=f(a)-a f^{\prime}(a)$ and $R=1-\delta+(1-\tau) r$ are constant;

(S2) $R=1 / \beta_{1}<1 / \beta_{2} \leq \ldots \leq 1 / \beta_{H}$;

(S3) $k=k_{1}>0$ and $k_{i}=0$ for $i \geq 2$;

(S4) $u_{1 c}\left(c_{1}, G\right)(1-\tau) w \leq v_{1}^{\prime}\left(l_{1}\right)$ and, for $i \geq 2, u_{i c}\left(c_{i}, G\right)(1-\tau) w=v_{i}^{\prime}\left(l_{i}\right)$;

(S5) $c_{1}=(R-1) k_{1}+(1-\tau) w l_{1}$ and $c_{i}=(1-\tau) w l_{i}$ for $i \geq 2$; 
(S6) $l=\sum_{i=1}^{H} l_{i}$;

(S7) $G=\tau\left(r k_{1}+\sum_{i=1}^{H} w l_{i}\right)=\tau f\left(k_{1} / l\right) l$.

Proof. See Appendix 7.1.

This proposition shows that because of the borrowing constraints, there exists a steady state. In accordance with the Ramsey (1928) conjecture and the seminal contribution of Becker (1980), the most patient household holds the whole capital stock. In contrast, in the absence of borrowing constraint, no steady state would exist (Le Van and Vailakis (2003), Le Van et al. (2007)). In this case indeed, the impatient agents reduce their consumption and increase their labor through time to reimburse their debt.

It is important to note that Proposition 1 suggests the existence of two types of steady state, one where the most patient household supplies no labor $\left(l_{1}=0\right)$ and one where all households are working $\left(0<l_{1}<\bar{\ell}\right)$. Indeed, since the most patient household, namely the capitalist, holds all the capital, he may choose not to work and share his after tax capital income between consumption and capital accumulation.

As already noticed by Bosi and Seegmuller (2010a), the steady state where the patient agent supplies no labor is undoubtedly of interest. In this case, two social classes emerge: the capitalist who smooths consumption on his infinite horizon and invests in productive capital, and $H-1$ workers who consume at each period their after-tax labor income. This property then provides foundations to models with population segmentation such as Mankiw (2000), Michel and Pestieau (1999) and Woodford (1986). In the following we focus exclusively on this type of steady state.

As preliminaries, let us define some useful elasticities. Households make their labor choice taking the prices $w$ and $r$ as given. Recall that the labor supply of impatient 
agents, $i=2, \ldots, H$, is defined by:

$$
u_{i c}\left((1-\tau) w l_{i}, G\right)(1-\tau) w=v_{i}^{\prime}\left(l_{i}\right)
$$

Using Assumption 1, this implicitly defines $l_{i}=l_{i}(w, G)$, where:

$$
\epsilon_{l_{i} G} \equiv \frac{d l_{i} / l_{i}}{d G / G}=\frac{\epsilon_{i c G}}{\epsilon_{i l l}+\epsilon_{i c c}}, \quad \epsilon_{l_{i} w} \equiv \frac{d l_{i} / l_{i}}{d w / w}=\frac{1-\epsilon_{i c c}}{\epsilon_{i l l}+\epsilon_{i c c}} \in(-1,+\infty)
$$

Using $\lambda_{i} \equiv l_{i} / l \in(0,1)$, we denote $\bar{\epsilon}_{l G} \equiv \sum_{i=2}^{H} \lambda_{i} \epsilon_{l_{i} G}$ and $\bar{\epsilon}_{l w} \equiv \sum_{i=2}^{H} \lambda_{i} \epsilon_{l_{i} w} \in(-1,+\infty)$.

We then derive the following existence result:

Corollary 1. Let Assumptions 1 and 2 hold, $\theta \equiv 1-\beta_{1}(1-\delta)$, a be the solution of $r(a)=\theta /\left(\beta_{1}(1-\tau)\right)$, and $c_{1}\left(l_{1}\right)$ and $l\left(l_{1}\right)$ be the capitalist's consumption and aggregate labor supply as functions of $l_{1} \cdot{ }^{10}$ There exists a steady state where the capitalist supplies no labor $\left(l_{1}=0\right)$ if and only if

$$
v_{1}^{\prime}(0) \geqslant u_{1 c}\left[c_{1}(0), \tau f(a) l(0)\right](1-\tau) w
$$

Proof. See Appendix 7.2.

If the capitalist's marginal disutility of labor is sufficiently large with respect to the marginal utility of consumption, he chooses not to work. The larger the capital stock at the steady state, the larger the capitalist's consumption, the lower his marginal utility of consumption and thus the more likely this case is. However, this effect can be mitigated by the public spending externality, if it positively affects the marginal utility of consumption. Indeed, a larger capital stock is associated with a higher total labor supply and may thus generate a larger capitalist's marginal utility of consumption. In the next section, we focus on the analysis of the equilibrium dynamics in the neighborhood of such a steady state.

\footnotetext{
${ }^{10}$ See Appendix 7.2 for formal definitions.
} 


\section{Endogenous business cycles under public spending}

\section{externalities}

Our aim is to show that, since public spending externalities affect utility of consumption, endogenous business cycles and expectation-driven fluctuations occur in the Ramsey model with heterogeneous agents when the capital-labor substitution is large enough. Such a conclusion is at odds with previous contributions studying this type of model (Becker and Foias (1987, 1994), Bosi and Seegmuller (2010a,b)). Moreover, it is worth pointing out that our results are obtained without introducing productive externalities and are compatible with a small elasticity of labor with respect to the wage rate.

As we focus on dynamics around the steady state without capitalist's labor supply $\left(l_{1}=0\right)$, we assume in the following that inequality (12) holds (see Corollary 1 ). To analyze the stability properties and the occurrence of endogenous cycles, we start by defining some additional useful elasticities, all evaluated at the steady state.

Taking into account that $w_{t}=w\left(k_{t} / l_{t}\right)$ and $G_{t}=\tau f\left(k_{t} / l_{t}\right) l_{t}$, the aggregate labor supply is given by:

$$
l_{t}=\sum_{i=2}^{H} l_{i}\left(w\left(k_{t} / l_{t}\right), \tau f\left(k_{t} / l_{t}\right) l_{t}\right)
$$

This expression implicitly defines $l_{t}=l\left(k_{t}\right)$, where $l^{\prime}(k) k / l(k) \equiv \epsilon_{l k}$ is given by:

$$
\epsilon_{l k}=\frac{\bar{\epsilon}_{l w} s / \sigma+\bar{\epsilon}_{l G} s}{1+\bar{\epsilon}_{l w} s / \sigma-\bar{\epsilon}_{l G}(1-s)}
$$

as long as $1+\bar{\epsilon}_{l w} s / \sigma-\bar{\epsilon}_{l G}(1-s) \neq 0$.

Using this result, in the neighborhood of the steady state exhibited in Corollary 1, the intertemporal equilibrium can be summarized by a two-dimensional dynamical system given by the patient household's trade-off between present and future consumption and 
his budget constraint. ${ }^{11}$ Indeed, using (10), (D8), (D7), (D6) and (13), an intertemporal equilibrium can be redefined as a sequence $\left(c_{1 t}, k_{t}\right)_{t=0}^{\infty}$, satisfying:

$$
\begin{aligned}
\frac{u_{1 c}\left[c_{1 t}, \tau f\left(k_{t} / l\left(k_{t}\right)\right) l\left(k_{t}\right)\right]}{u_{1 c}\left[c_{1 t+1}, \tau f\left(k_{t+1} / l\left(k_{t+1}\right)\right) l\left(k_{t+1}\right)\right]} & =\beta_{1} R\left(k_{t+1} / l\left(k_{t+1}\right)\right) \\
k_{t+1} & =R\left(k_{t} / l\left(k_{t}\right)\right) k_{t}-c_{1 t}
\end{aligned}
$$

where $l\left(k_{t}\right)$ is defined by $(13), c_{1 t}$ is a forward variable and $k_{t}$ is the only predetermined variable.

We characterize the stability properties of the steady state and the occurrence of local bifurcations by linearizing the dynamic system (15)-(16) around the steady state $\left(c_{1}, k\right)$ and computing the Jacobian matrix $J$, evaluated at this steady state. Following Grandmont et al. (1998), we compute the trace $T$ and the determinant $D$ of the associated Jacobian matrix $J$ and study the stability properties by locating them in the $(T, D)$-plane (see Figures 1-2).

Lemma 1. Under Assumptions 1 and 2, the characteristic polynomial is given by $P(x) \equiv$ $x^{2}-T x+D=0$, where:

$$
\begin{aligned}
T & =1+\frac{1}{\beta_{1}}+\theta(1-s) \frac{1-\bar{\epsilon}_{l G}}{\sigma\left(1-(1-s) \bar{\epsilon}_{l G}\right)+s \bar{\epsilon}_{l w}} \frac{1-\beta_{1}-\epsilon_{1 c c}}{\beta_{1} \epsilon_{1 c c}} \\
& -\frac{1-\beta_{1}}{\beta_{1}} \frac{\epsilon_{1 c G}}{\epsilon_{1 c c}} s \frac{\sigma+\bar{\epsilon}_{l w}}{\sigma\left(1-(1-s) \bar{\epsilon}_{l G}\right)+s \bar{\epsilon}_{l w}} \equiv T(\sigma) \\
D & =T-1-\theta(1-s) \frac{1-\bar{\epsilon}_{l G}}{\sigma\left(1-(1-s) \bar{\epsilon}_{l G}\right)+s \bar{\epsilon}_{l w}} \frac{1-\beta_{1}}{\beta_{1} \epsilon_{1 c c}} \equiv D(\sigma)
\end{aligned}
$$

Proof. See Appendix 7.3.

To apply the geometrical method developped by Grandmont et al. (1998), we choose $\sigma \geq 0$ as the bifurcation parameter. This choice is especially relevant regarding previous

\footnotetext{
${ }^{11}$ The dynamic path requires $u_{i c}\left(w_{t} l_{i t}, G_{t}\right)>\beta_{i} R_{t+1} u_{i c}\left(w_{t+1} l_{i t+1}, G_{t+1}\right)$, for all $i \geq 2$, which can be ensured by a sufficiently low discount factor $\beta_{i}$.
} 
contributions showing that endogenous fluctuations can occur in Ramsey models with heterogeneous consumers (Becker and Foias (1987, 1994), Bosi and Seegmuller (2010a)). A key result of this literature is that endogenous cycles require a low enough elasticity of capital-labor substitution, allowing the capital income to decrease with respect to capital $\left(\sigma<(1-s)\left[1-\beta_{1}(1-\delta)\right]\right)$. Such a condition appears to be extremely restrictive and not compatible with empirically realistic values for $\sigma$ (see footnote 2 ).

Considering now the $(T, D)$-plane, following a variation of the elasticity of capitallabor substitution $\sigma$, the locus $\Sigma \equiv\{(T(\sigma), D(\sigma)): \sigma \geq 0\}$ describes an (unconnected) half-line, with endpoint:

$$
\begin{aligned}
T(+\infty) & =1+\frac{1}{\beta_{1}}-\frac{1-\beta_{1}}{\beta_{1}} \frac{\epsilon_{1 c G}}{\epsilon_{1 c c}} \frac{s}{1-(1-s) \bar{\epsilon}_{l G}} \\
D(+\infty) & =T(+\infty)-1
\end{aligned}
$$

and a slope $S=D^{\prime}(\sigma) / T^{\prime}(\sigma)$ given by:

$$
S=\frac{\theta\left(1-(1-s) \bar{\epsilon}_{l G}\right) \epsilon_{1 c c}+\left(1-\beta_{1}\right) \epsilon_{1 c G} s \bar{\epsilon}_{l w}}{\theta\left(1-(1-s) \bar{\epsilon}_{l G}\right)\left(\epsilon_{1 c c}-1+\beta_{1}\right)+\left(1-\beta_{1}\right) \epsilon_{1 c G} s \bar{\epsilon}_{l w}}
$$

To present our results using the geometrical method introduced by Grandmont et al. (1998), we start by considering the case where the labor supply is inelastic with respect to the wage, but still depends on public spending, i.e. $\bar{\epsilon}_{l w}=0$. Note that this implicitly means that $\epsilon_{i c c}=1$ (see equation (11)). In a second step, we will generalize this analysis to the case where the labor supply depends on the real wage.

By direct inspection of equations (17) and (18), we see that $T(0)= \pm \infty$ and $D(0)=$ $\pm \infty$, depending on the slope $S$, which becomes:

$$
S=\frac{\epsilon_{1 c c}}{\epsilon_{1 c c}-\left(1-\beta_{1}\right)}
$$

It follows that $S$ is decreasing in $\epsilon_{1 c c}$, with $S>1$ for all $\epsilon_{1 c c}>1-\beta_{1}, S \in(-\infty, 0)$ for $\epsilon_{1 c c}<1-\beta_{1}$ and $S \rightarrow 0$ when $\epsilon_{1 c c} \rightarrow 0$. As depicted in Figure 1, when $\epsilon_{1 c c}$ decreases, $\Sigma$ makes a counterclockwise rotation around $(T(+\infty), D(+\infty))$, which is located on the 
$(A C)$ line, becoming flat when $\epsilon_{1 c c} \rightarrow 0$. Therefore, $D(+\infty)<1$ and $D^{\prime}(\sigma)<0$ are two necessary conditions to get indeterminacy and Hopf bifurcation.

We have that $D(+\infty)<1$ if $\bar{\epsilon}_{l G}<1 /(1-s)$ and $\epsilon_{1 c G}>\epsilon_{1 c G}^{\min }$, with:

$$
\epsilon_{1 c G}^{\min } \equiv \frac{\epsilon_{1 c c}}{s}\left[1-(1-s) \bar{\epsilon}_{l G}\right]
$$

Taking into account that $\beta_{1}$ is usually assumed to be close to 1 , we have $D(+\infty)>-1$ for reasonable levels of public spending externalities, i.e. $\epsilon_{1 c G}<\epsilon_{1 c G}^{\max }$, with:

$$
\epsilon_{1 c G}^{\max } \equiv \frac{1+\beta_{1}}{1-\beta_{1}} \frac{\epsilon_{1 c c}}{s}\left[1-(1-s) \bar{\epsilon}_{l G}\right]
$$

Using equation (18), we also get that when $\bar{\epsilon}_{l w}=0$ and $\bar{\epsilon}_{l G}<1 /(1-s), D^{\prime}(\sigma)<0$ if and only if $\bar{\epsilon}_{l G}>1$. Let us then assume:

Assumption 3. $\epsilon_{1 c G} \in\left(\epsilon_{1 c G}^{\min }, \epsilon_{1 c G}^{\max }\right)$ and $\bar{\epsilon}_{l G} \in(1,1 /(1-s))$.

This means that public spending positively affects the marginal utility of consumption for the patient agent and the impatient ones (see equation (11)), but this effect should not be too large.

Let us define $\sigma_{H}$ such that one crosses the segment $[B C], \sigma_{F}$ such that one crosses the line $(A B), \epsilon_{1 c c}^{F}$ such that $S=-1$ and $\epsilon_{1 c c}^{H}$ such that $\Sigma$ goes through the point $B .{ }^{12}$ Using our geometrical arguments and as drawn in Figure 1, we conclude that when $\epsilon_{1 c c}>\epsilon_{1 c c}^{F}$, $(T, D)$ is above $(A B),(A C)$ and $[B C]$ for $\sigma<\sigma_{H}$, crosses $[B C]$ for $\sigma=\sigma_{H}$ and is inside $A B C$ for $\sigma>\sigma_{H}$. When $\epsilon_{1 c c}^{H}<\epsilon_{1 c c}<\epsilon_{1 c c}^{F},(T, D)$ is on the left-side of $(A B)$ for $\sigma<\sigma_{F}$, crosses $(A B)$ for $\sigma=\sigma_{F}$, is above $(A B),(A C)$ and $[B C]$ for $\sigma_{F}<\sigma<\sigma_{H}$, crosses $[B C]$ for $\sigma=\sigma_{H}$ and is inside $A B C$ for $\sigma>\sigma_{H}$. Finally, when $\epsilon_{1 c c}<\epsilon_{1 c c}^{H},(T, D)$ is on the left-side of $(A B)$ for $\sigma<\sigma_{F}$, crosses $(A B)$ for $\sigma=\sigma_{F}$ and is inside $A B C$ for $\sigma>\sigma_{F}$.

We then get the following Lemma:

\footnotetext{
${ }^{12}$ The critical values $\sigma_{H}, \sigma_{F}, \epsilon_{1 c c}^{F}$ and $\epsilon_{1 c c}^{H}$ are given in Appendix 7.4.
} 




Figure 1: Local indeterminacy when $\bar{\epsilon}_{l w}=0$

Lemma 2. Under Assumptions 1-3 and $\bar{\epsilon}_{l w}=0$, indeterminacy occurs if either $\epsilon_{1 c c}>\epsilon_{1 c c}^{H}$ and $\sigma>\sigma_{H}$, or $\epsilon_{1 c c}<\epsilon_{1 c c}^{H}$ and $\sigma>\sigma_{F}$. Moreover, a Hopf bifurcation generically occurs when $\sigma$ crosses $\sigma_{H}$ while a flip bifurcation generically occurs when when $\sigma$ crosses $\sigma_{F}$.

Using these preliminary results, we generalize now our analysis to an elastic labor supply with respect to the real wage, i.e. $\bar{\epsilon}_{l w} \neq 0$. We consider the case $\bar{\epsilon}_{l w}=0$ as a benchmark and analyze what changes when $\bar{\epsilon}_{l w}$ deviates from 0 . We observe that the endpoint $(T(+\infty), D(+\infty))$ does not depend on $\bar{\epsilon}_{l w}$, i.e. stays on the $(A C)$ line. Moreover, using (21), we derive:

$$
\frac{\partial S}{\partial \bar{\epsilon}_{l w}}<0 \text { and } \lim _{\bar{\epsilon}_{l w} \rightarrow+\infty} S=1
$$

Let $\bar{\epsilon}_{l w}^{*}$ be defined by $S=0$ :

$$
\bar{\epsilon}_{l w}^{*} \equiv-\frac{\theta\left[1-(1-s) \bar{\epsilon}_{l G}\right] \epsilon_{1 c c}}{\left(1-\beta_{1}\right) \epsilon_{1 c G} s}<0
$$

Taking into account that $\bar{\epsilon}_{l w} \in(-1,+\infty)$ and $\bar{\epsilon}_{l w}^{*}$ can be larger than -1 , we assume 
in the following that:

Assumption 4. $\bar{\epsilon}_{l w}>\bar{\epsilon}_{l w}^{*}$.

First, this is satisfied if public spending externalities are not too large, i.e. $\epsilon_{1 c G}<\theta[1-$ $\left.(1-s) \bar{\epsilon}_{l G}\right] \epsilon_{1 c c} /\left[s\left(1-\beta_{1}\right)\right] .{ }^{13}$ Indeed, in this case, $\bar{\epsilon}_{l w}^{*}<-1$. Second, if this last inequality is not fulfilled, $\bar{\epsilon}_{l w}>\bar{\epsilon}_{l w}^{*}$ is ensured by assuming that the elasticity of intertemporal substitution in consumption of the impatient agents is not too low, which is in accordance with the recent empirical evidence. ${ }^{14}$ Consider that all impatient agents are identical to household $i=2$. Using (11), we have $\bar{\epsilon}_{l w}=\left(1-\epsilon_{2 c c}\right) /\left(\epsilon_{2 l l}+\epsilon_{2 c c}\right)$. Therefore, $\bar{\epsilon}_{l w}>\bar{\epsilon}_{l w}^{*}$ is equivalent to $\epsilon_{2 c c}<\left(1-\bar{\epsilon}_{l w}^{*} \epsilon_{2 l l}\right) /\left(1+\bar{\epsilon}_{l w}^{*}\right)$. Taking into account that the right-hand side of this inequality is sufficiently high, in any case larger than 1 , this requires that the elasticity of intertemporal substitution in consumption $1 / \epsilon_{2 c c}$ should not be too weak.

Our strategy is to see what happens when $\bar{\epsilon}_{l w}$ deviates from 0, keeping the other parameters as given. Using (25), we immediately conclude that $\Sigma$ makes a counterclockwise rotation around $(T(+\infty), D(+\infty))$ when $\bar{\epsilon}_{l w}$ decreases from $+\infty$ to $\bar{\epsilon}_{l w}^{*}$. More precisely, $S$ increases from 1 to $+\infty$ and, then, from $-\infty$ to 0 . This means that we have either $S>1$ or $S<0$. Therefore, the geometrical configurations are similar to the preliminary case where $\bar{\epsilon}_{l w}=0$, except that we will now classify them according to the value of $\bar{\epsilon}_{l w}$ (see also Figure 2).

\footnotetext{
${ }^{13}$ Note that the right-hand side of this inequality is larger than $\epsilon_{1 c G}^{\min }$.

${ }^{14}$ The elasticity of intertemporal substitution in consumption is usually assumed to be less than one. However, the recent contributions provide divergent views. Mulligan [24], Vissing-Jorgensen [29] and Vissing-Jorgensen and Attanasio [30] repeatedly obtained estimates of this elasticity which are significantly larger than one. More recently, Gruber [15] and Kapoor and Ravi [17] provide robust estimates in the range $(2,3)$.
} 
As a direct implication, two necessary conditions to get indeterminacy and Hopf bifurcation are $D(+\infty)<1$ and $D^{\prime}(\sigma)<0$. The first one is ensured by Assumption 3, while the second condition is satisfied under Assumptions 3-4.

Let us define $\bar{\epsilon}_{l w}^{F}$ such that $S=-1$ and $\bar{\epsilon}_{l w}^{H}$ such that $\Sigma$ goes through the point $B$. We can use the same geometrical arguments than in the case $\bar{\epsilon}_{l w}=0$ to get the results of the following proposition (see also Figure 2). Note however that when $\bar{\epsilon}_{l w} \neq 0, T(0)$ and $D(0)$ take now finite values. Using (17) and (18), we can easily show that $(T(0), D(0))$ is on the right-side of $(A C)$ when $\bar{\epsilon}_{l w}<0$, whereas it is located above $(A C)$ when $\bar{\epsilon}_{l w}>0$. In this last case, the critical values $\sigma_{H}$ and $\sigma_{F}$ can be positive or negative depending on parameter configurations. ${ }^{15}$

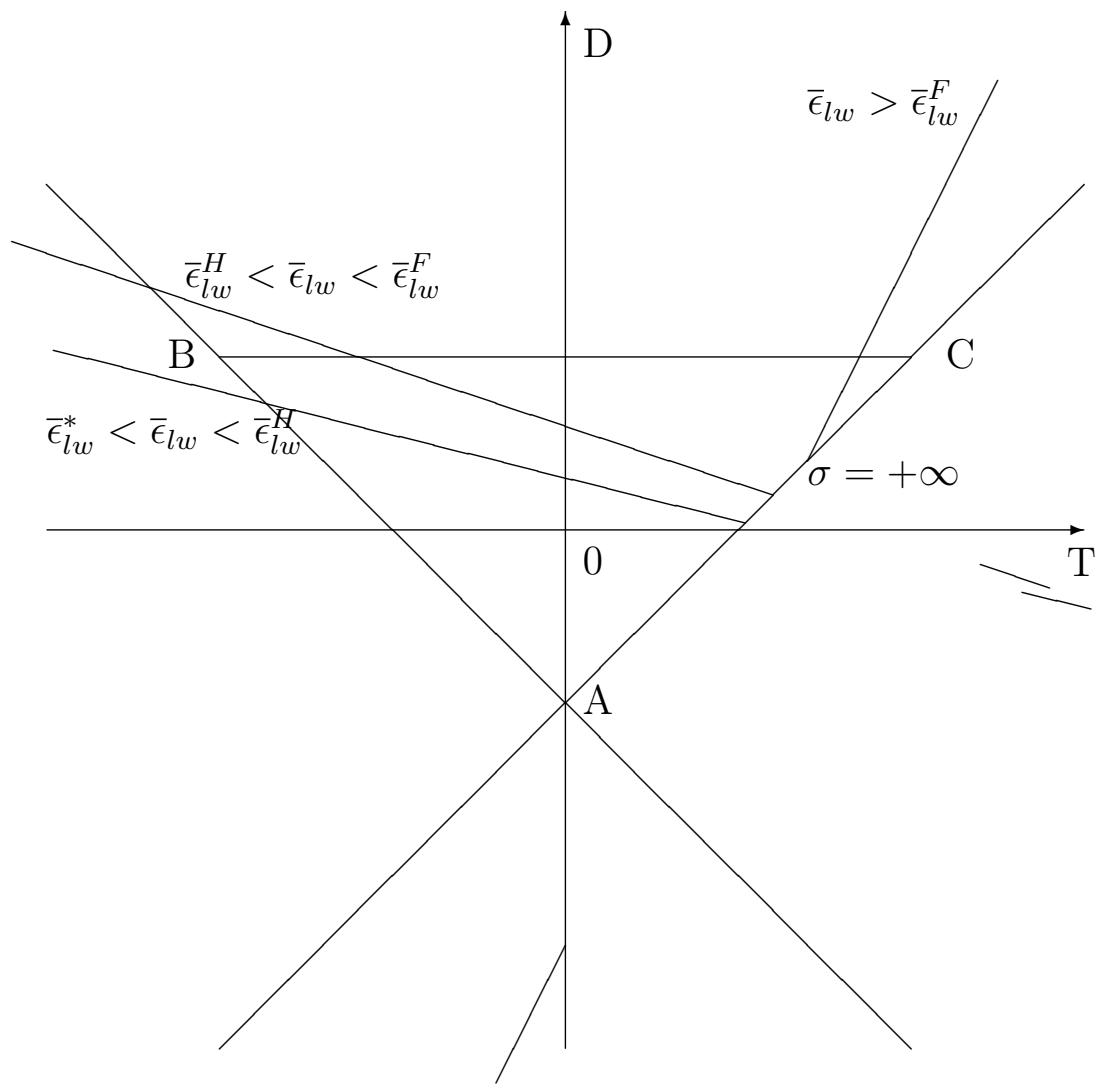

Figure 2: Local indeterminacy when $\bar{\epsilon}_{l w}>\bar{\epsilon}_{l w}^{*}$

\footnotetext{
${ }^{15}$ The critical values $\bar{\epsilon}_{l w}^{F}, \bar{\epsilon}_{l w}^{H}, \sigma_{H}$ and $\sigma_{F}$ are given in Appendix 7.4.
} 
We then get the following Proposition:

Proposition 2. Under Assumptions 1-4, the following results generically hold:

(i) When $\bar{\epsilon}_{l w}>\bar{\epsilon}_{l w}^{F}$, the steady state is unstable for $\sigma<\sigma_{H}$, undergoes a Hopf bifurcation for $\sigma=\sigma_{H}$, and becomes locally indeterminate (stable) for $\sigma>\sigma_{H}$;

(ii) When $\bar{\epsilon}_{l w}^{H}<\bar{\epsilon}_{l w}<\bar{\epsilon}_{l w}^{F}$, the steady state is a saddle for $\sigma<\sigma_{F}$, undergoes a flip bifurcation for $\sigma=\sigma_{F}$, is unstable for $\sigma_{F}<\sigma<\sigma_{H}$, undergoes a Hopf bifurcation for $\sigma=\sigma_{H}$, and becomes locally indeterminate (stable) for $\sigma>\sigma_{H}$;

(iii) When $\bar{\epsilon}_{l w}^{*}<\bar{\epsilon}_{l w}<\bar{\epsilon}_{l w}^{H}$, the steady state is a saddle for $\sigma<\sigma_{F}$, undergoes a flip bifurcation for $\sigma=\sigma_{F}$, and becomes locally indeterminate (stable) for $\sigma>\sigma_{F}$.

This proposition shows that endogenous business cycles emerge through Hopf or flip bifurcations, leading to expectation-driven fluctuations for $\sigma>\max \left\{\sigma_{H}, \sigma_{F}\right\}$. In contrast to previous contributions focusing on Ramsey models with heterogeneous households, endogenous fluctuations occur for a large range of elasticities of capital-labor substitution including high values. This is at odds with the non monotonicity of capital income required to get period-two cycles, in the model with neither endogenous labor, nor public spending externalities (Becker and Foias $(1987,1994))$. Note that this last condition can be slightly relaxed when elastic labor is introduced (Bosi and Seegmuller (2010a)). However, in all these papers, local indeterminacy and Hopf bifurcations are ruled out. Expectation-driven fluctuations can be obtained if non-linear tax rates are introduced, but this still requires a weak input substitutability (Bosi and Seegmuller (2010b)).

Let us emphasize that limit cycles occur for the more relevant parameter configurations. If one considers as usual the discount factor $\beta_{1}$ close to 1 , reasonable or not too 
large values of the elasticity of intertemporal substitution in consumption $1 / \epsilon_{1 c c}$ and of the wage elasticity of labor supply $\bar{\epsilon}_{l w}$, configuration $(i)$ of Proposition 2 is the most relevant one. ${ }^{16}$ In this case, local indeterminacy occurs for $\sigma>\sigma_{H}$ and endogenous cycles appear through a Hopf bifurcation.

A numerical illustration: Let us consider a simple example in order to show that our conditions for indeterminacy are compatible with plausible parameterizations of the underlying economy. We consider the following fundamentals:

$$
u_{i}\left(c_{i}, G\right)=\frac{c_{i}^{1-\theta_{i}}}{1-\theta_{i}} G^{\gamma_{i}}, v_{i}\left(l_{i}\right)=-B \frac{\left(\bar{\ell}-l_{i}\right)^{1-\chi_{i}}}{1-\chi_{i}} \text { and } f(a)=A\left[\eta a^{-\rho}+1-\eta\right]^{-1 / \rho}
$$

Beside agent 1 , we assume that all the other agents $i=2, \ldots, H$ are identical. To fit quarterly data, we set the depreciation rate of capital to $\delta=0.025$, the subjective discount factors to $\beta_{1}=0.99, \beta_{i}=0.98$, and the capital share of national income to $\eta=0.3$. Based on our reading of the recent empirical literature (see footnotes 5 and 14), we consider the following parameters' values: $\chi_{1}=0.4, \chi_{i}=0.35, \theta_{1}=0.52, \theta_{i}=0.34$. Concerning the size of the public spending externality, ${ }^{17}$ we set $\gamma_{1}=0.55$ and $\gamma_{i}=0.6905$. All the Assumptions are satisfied since $\gamma_{1}>\theta_{1}, \chi_{i}+\theta_{i}<\gamma_{i}<\left(\chi_{i}+\theta_{i}\right) /(1-\eta)$ and $\theta_{1}, \theta_{i}<1$, and condition (12) of Corollary 1 holds. We derive that local indeterminacy arises with complex roots for any elasticity of capital-labor substitution $\sigma=1 /(1+\rho)$ within the interval $[0.4,3.24]$ of plausible values (see footnote 2$).{ }^{18}$

Previous contributions have already stressed the role of public spending on the oc-

\footnotetext{
${ }^{16}$ Indeed, we have $\epsilon_{1 c c}>\left(1-\beta_{1}\right) / 2$ and $\bar{\epsilon}_{l w}^{F}<0$.

${ }^{17} \mathrm{Ni}[25]$ provides estimates of a similar Cobb-Douglas utility function and shows that the parameter $\gamma$ lies in the interval $(0.5,0.8)$.

${ }^{18}$ Details of the numerical simulations are available upon request.
} 
currence of local indeterminacy, especially in models with representative agents. But, in contrast to our result, deterministic cycles do not appear in the absence of productive externalities. Actually, expectation-driven fluctuations occur if households' utility displays increasing returns to scale in private consumption and public spending (Cazzavillan (1996), Guo and Harrison (2008), Zhang (2000)). We can show that in our framework, such a property is not necessary for all the agents.

If $u_{i}\left(c_{i}, G\right)$ is homogeneous of degree $\mu$, increasing returns mean that $\mu>1$, or equivalently $\epsilon_{i c G}>\epsilon_{i c c}$. A first necessary condition for endogenous fluctuations when capital and labor are substitutes is $\bar{\epsilon}_{l G}>1$. To simplify, if all impatient workers are similar to household $i=2$, this can be rewritten as $\epsilon_{2 c G}>\epsilon_{2 c c}+\epsilon_{2 l l}$, which precisely ensures increasing returns in utility for these agents. A second necessary condition is $\epsilon_{1 c G}>\epsilon_{1 c G}^{m i n}$. Since $\bar{\epsilon}_{l G}>1$, we derive from $(23)$ that $\epsilon_{1 c G}^{m i n}<\epsilon_{1 c c}$. Therefore, if $\epsilon_{1 c G}$ belongs to $\left(\epsilon_{1 c G}^{m i n}, \epsilon_{1 c c}\right)$, indeterminacy is compatible with decreasing returns in the capitalist's utility.

It is important to note that endogenous labor plays a crucial role for the existence of expectation-driven fluctuations, through the link between labor supply and public spending. Indeed, without endogenous labor, we have $\bar{\epsilon}_{l G}=\bar{\epsilon}_{l w}=0$, which implies that the steady state cannot be indeterminate and Hopf bifurcations never occur. ${ }^{19}$ However, if public spending externalities are large enough for workers $\left(\bar{\epsilon}_{l G}>1\right)$, expectation-driven fluctuations and endogenous business cycles occur under a weakly elastic or even inelastic labor supply with respect to wage. This is in contrast to models with public spending externalities and a representative consumer where an infinitely elastic labor supply is often

\footnotetext{
${ }^{19}$ Indeed, using our geometrical arguments, $D^{\prime}(\sigma)>0$ with $S>1$ or $S<0$ and $D(0)=-\infty$, meaning that $\Sigma$ never enters $A B C$.
} 
assumed (see for instance Guo and Harrison (2008)).

Let us finally examine how the two critical values $\sigma_{H}$ and $\sigma_{F}$ evolve according to the elasticity of labor supply with respect to wage. Using the expressions found in Appendix 7.4, we observe that $\sigma_{H}$ is increasing in $\bar{\epsilon}_{l w}$ if and only if $\epsilon_{1 c G}<1$, while $\sigma_{F}$ is increasing in $\bar{\epsilon}_{l w}$ if and only if $\epsilon_{1 c G}>\epsilon_{1 c c}\left(1+\beta_{1}\right) /\left(1-\beta_{1}\right)$. As seen above, for reasonable parameter values, $\sigma_{H}$ seems to be the relevant bifurcation value above which indeterminacy occurs. Therefore, if the public spending externality on capitalist's utility is not too large $\left(\epsilon_{1 c G}<1\right)$, larger wage elasticities of workers' labor supplies stabilize, reducing the range of elasticities of input substitution for indeterminacy. In contrast, if the public spending externality on capitalist's utility is sufficiently large $\left(\epsilon_{1 c G}>1\right)$, we get the opposite conclusion.

\section{Economic intuition}

In Ramsey models with heterogeneous households, the interpretation of cycles is usually linked to the capitalist's budget constraint. Indeed, fluctuations occur if the capital income monotonicity fails, i.e. for sufficiently low degrees of capital-labor substitution $(\sigma<$ $\left.(1-s)\left[1-\beta_{1}(1-\delta)\right]\right)$ (see Becker and Foias $\left.(1987,1994)\right)$. As mentioned previously, such a condition appears to be extremely restrictive and thus not compatible with empirically realistic values for $\sigma$.

In our framework, endogenous cycles also occur under a large elasticity of capital-labor substitution, in particular such that $\sigma>(1-s)\left[1-\beta_{1}(1-\delta)\right]$. Obviously, this means that the standard mechanism based on the capitalist's budget constraint does not allow to generate fluctuations. But this also suggests that the explanation of expectation-driven 
fluctuations actually relies on an alternative mechanism which is rather mainly based on the Euler equation:

with

$$
\frac{u_{1 c}\left(c_{1 t}, G_{t}\right)}{u_{1 c}\left(c_{1 t+1}, G_{t+1}\right)}=\beta_{1} R_{t+1}
$$

$$
G_{t}=\tau f\left(k_{t} / l_{t}\right) l_{t}
$$

To simplify, let us focus on the case where the labor supply is inelastic with respect to the wage rate $\left(\bar{\epsilon}_{l w}=0\right)$. We derive from equation $(11)$ that this case is obtained if $\epsilon_{i c c}=1$ for any $i \geqslant 2$.

Let households be optimistic and the capitalist deviates from the steady state by investing more, i.e. $k_{t+1}$ increases. This means that both current consumption $c_{1 t}$ and the return on capital $R_{t+1}$ decreases, but since capital and labor are high substitutes $\left(\sigma>(1-s)\left[1-\beta_{1}(1-\delta)\right]\right)$, the capitalist's income $R_{t+1} k_{t+1}$ increases in the next period. This implies a raise of future consumption $c_{1 t+1}$. Therefore, for a constant level of public spending, the right-hand side of equation (27) decreases, while the left-hand side increases because of both the raise of $c_{1 t+1}$ and the lower $c_{1 t}$. This excludes self-fulfilling fluctuations.

However, considering the impact of public spending on the marginal utility of consumption can yield an additional effect which mitigates the increase of the left-hand side. It will also induce an increase of the return on capital.

Indeed, the rise of $k_{t+1}$ now implies a variation of public spending $G_{t+1}$, which generates an increase of the marginal utility of consumption at $t+1$. As labor supply depends on public spending, $G_{t}$ can be rewritten as a function of capital. Using (28), we get:

$$
\epsilon_{G k} \equiv \frac{d G / G}{d k / k}=\frac{s}{1-(1-s) \bar{\epsilon}_{l G}}
$$

Based on this expression, and considering that since $\bar{\epsilon}_{l w}=0$, we have $d k_{t+1} / k=d c_{1 t+1 /} c_{1}$. It follows that the marginal utility of consumption at $t+1$ increases if

$$
\epsilon_{1 c G} \epsilon_{G k} \frac{d k_{t+1}}{k}-\epsilon_{1 c c} \frac{d c_{1 t+1}}{c_{1}}=\frac{d k_{t+1}}{k}\left[\epsilon_{1 c G} \frac{s}{1-(1-s) \bar{\epsilon}_{l G}}-\epsilon_{1 c c}\right]>0
$$


Using equation (23) we derive that this condition is equivalent to $\epsilon_{1 c G}>\epsilon_{1 c G}^{\min }$. Recall then from Proposition 2 that this is a necessary condition to have endogenous business cycles when $\sigma$ is large enough. This ensures that the increase of the left-hand side of equation (27), due to a lower $c_{1 t}$, is mitigated.

The second important point is to notice that under $1<\bar{\epsilon}_{l G}<1 /(1-s)$, we have $\epsilon_{l k}>1$ (see equation (14)). ${ }^{20}$ This means that the capital-labor ratio decreases with respect to $k$. Therefore, following an increase of $k_{t+1}$, the return on capital $R_{t+1}$ raises, slightly when capital and labor are sufficiently substitutable. Therefore, the rise of $k_{t+1}$ now implies an increase of both the left and right-hand side of equation (27) so that the expectations are self-fulfilling.

Note that this intuition stresses the important role played by the labor market, since it allows the return of capital to increase with capital. The role of the labor market on the occurrence of indeterminacy is however different from most one-sector models with a representative household. Indeed, in our framework with many agents, impatient workers make a static trade-off between consumption and leisure, because of their binding borrowing constraints. Therefore, labor supply does not depend on expectations on future prices.

\section{Conclusion}

Public spending is introduced in the Ramsey model with heterogeneous households developed by Becker (1980) and recently extended to endogenous labor by Bosi and Seegmuller (2010a). They are financed through a linear taxation on income and positively

\footnotetext{
${ }^{20}$ We thank a referee to underline this point.
} 
affect households' welfare. The existence of such an externality is a source of endogenous business cycles. Indeed, in contrast to previous contributions focusing on Ramsey models with heterogeneous agents, endogenous fluctuations emerge for large enough degrees of capital-labor substitution. Another new contribution to this literature is that endogenous cycles occur around the steady state through a Hopf bifurcation, underlying the persistence of fluctuations. Finally, in contrast to models with a representative agent, when heterogeneous households are considered, endogenous business cycles do not require productive externalities and are in accordance with a weakly elastic labor supply with respect to the wage rate.

\section{Appendix}

\subsection{Proof of Proposition 1}

The proof of this proposition consists in three steps.

Step 1. For $i=1$, (S2)-(S5) satisfy the optimality conditions in Definition 1. Moreover, since $c_{1}, k_{1}$ and $G$ are constant and $0<\beta_{1}<1$, the transversality condition $\lim _{t \rightarrow+\infty} \beta_{1}^{t} u_{1 c}\left(c_{1}, G\right) k_{1}=0$ holds.

Step 2. For $i \geq 2$, given $G$, consider the feasible sequence $\left(\tilde{k}_{i t}, \tilde{l}_{i t}, \tilde{c}_{i t}\right)$, starting from $\tilde{k}_{i 0}=0$. We now compare this path with the stationary solution $\left(c_{i}, l_{i}\right)$, such that $k_{i}=0$, $1>l_{i}>0$ and $c_{i}=(1-\tau) w l_{i}$, and show that the stationary solution is optimal. 


$$
\begin{aligned}
& \sum_{t=0}^{+\infty} \beta_{i}^{t}\left[u_{i}\left(c_{i}, G\right)-v_{i}\left(l_{i}\right)-\left(u_{i}\left(\tilde{c}_{i t}, G\right)-v_{i}\left(\tilde{l}_{i t}\right)\right)\right] \\
\geq & \sum_{t=0}^{+\infty} \beta_{i}^{t}\left[u_{i c}\left((1-\tau) w l_{i}, G\right)\left((1-\tau) w l_{i}-\tilde{c}_{i t}\right)-v_{i}^{\prime}\left(l_{i}\right)\left(l_{i}-\tilde{l}_{i t}\right)\right] \\
= & u_{i c}\left((1-\tau) w l_{i}, G\right) \lim _{T \rightarrow+\infty}\left[\beta_{i}^{T} \tilde{k}_{i T+1}+\left(1 / \beta_{i}-1 / \beta_{1}\right) \sum_{t=1}^{T} \beta_{i}^{t} \tilde{k}_{i t}-\tilde{k}_{i 0} / \beta_{1}\right] \\
\geq & -u_{i c}\left((1-\tau) w l_{i}, G\right) \tilde{k}_{i 0} / \beta_{1}=0
\end{aligned}
$$

Step 3. Under Assumption 2, there is a unique finite and strictly positive value of a such that $R(a)=1-\delta+(1-\tau) f^{\prime}(a)=1 / \beta_{1}$. We further note that:

1. If $R>1 / \beta_{1}$, then it is optimal for the most patient household to increase capital. This cannot be a stationary solution because of decreasing returns in capital.

2. If $R<1 / \beta_{1}<1 / \beta_{2} \leq \ldots \leq 1 / \beta_{H}$, each household decumulates to zero. Since $l>0$, $a_{t}$ tends to 0 and $f^{\prime}\left(a_{t}\right)$ to $+\infty$, violating stationarity.

\subsection{Proof of Corollary 1}

Using $G=\tau f(a) l$, the aggregate labor supply is given by $l=l_{1}+\sum_{i=2}^{H} l_{i}(w, \tau f(a) l)$. This implicitly defines $l=l\left(l_{1}\right)$, with:

$$
\frac{d l}{d l_{1}}=\frac{1}{1-\bar{\epsilon}_{l G}}
$$

Consider now household $i=1$. At a steady state, his consumption is given by:

$$
c_{1}=\left(1 / \beta_{1}-1\right) k_{1}+(1-\tau) w l_{1}=(1-\tau) w l_{1}+\left(1 / \beta_{1}-1\right) a l\left(l_{1}\right) \equiv c_{1}\left(l_{1}\right)
$$

Then $l_{1}=0$ is a stationary solution iff $\lim _{l_{1} \rightarrow 0} \psi\left(l_{1}\right) \geq 0$, with:

$$
\psi\left(l_{1}\right) \equiv v_{1}^{\prime}\left(l_{1}\right)-u_{1 c}\left[c_{1}\left(l_{1}\right), \tau f(a) l\left(l_{1}\right)\right](1-\tau) w
$$

\subsection{Proof of Lemma 1}

Linearizing the dynamic system (15)-(16) around the steady state, we obtain: 


$$
\begin{aligned}
& \frac{d k_{t+1}}{k}=\frac{1}{\beta_{1}}\left[1-\theta \frac{1-s}{\sigma}\left(1-\epsilon_{l k}\right)\right] \frac{d k_{t}}{k}-\frac{1-\beta_{1}}{\beta_{1}} \frac{d c_{1 t}}{c_{1}} \\
& \frac{d c_{1 t+1}}{c_{1}}=\left\{-\frac{\epsilon_{1 c G}}{\epsilon_{1 c c}}\left(s+(1-s) \epsilon_{l k}\right)+\frac{1}{\beta_{1}}\left(1-\theta \frac{1-s}{\sigma}\left(1-\epsilon_{l k}\right)\right) \frac{1}{\epsilon_{1 c c}}\right. \\
& \left.\left[\epsilon_{1 c G}\left(s+(1-s) \epsilon_{l k}\right)-\theta \frac{1-s}{\sigma}\left(1-\epsilon_{l k}\right)\right]\right\} \frac{d k_{t}}{k} \\
& +\left\{1-\frac{1-\beta_{1}}{\beta_{1} \epsilon_{1 c c}}\left[\epsilon_{1 c G}\left(s+(1-s) \epsilon_{l k}\right)-\theta \frac{1-s}{\sigma}\left(1-\epsilon_{l k}\right)\right]\right\} \frac{d c_{1 t}}{c_{1}}
\end{aligned}
$$

Since $T$ and $D$ represent respectively the trace and the determinant of the associated

Jacobian matrix, we get:

$$
\begin{aligned}
T & =1+\frac{1}{\beta_{1}}+\theta \frac{1-s}{\sigma}\left(1-\epsilon_{l k}\right)\left(\frac{1-\beta_{1}}{\beta_{1} \epsilon_{1 c c}}-\frac{1}{\beta_{1}}\right)-\frac{1-\beta_{1}}{\beta_{1}} \frac{\epsilon_{1 c G}}{\epsilon_{1 c c}}\left(s+(1-s) \epsilon_{l k}\right) \\
D & =T-1-\theta \frac{1-s}{\sigma}\left(1-\epsilon_{l k}\right) \frac{1-\beta_{1}}{\beta_{1} \epsilon_{1 c c}}
\end{aligned}
$$

Substituting $\epsilon_{l k}$ given by equation (14) in these two expressions, the result follows.

\subsection{Critical values}

\section{Critical values $\sigma^{F}$ and $\sigma^{H}$}

The critical value $\sigma_{H}$ solves $D\left(\sigma_{H}\right)=1$ :

$$
\sigma_{H}=\frac{\theta(1-s)\left(\bar{\epsilon}_{l G}-1\right) \epsilon_{1 c c}+s\left(1-\beta_{1}\right)\left(\epsilon_{1 c c}-\epsilon_{1 c G}\right) \bar{\epsilon}_{l w}}{\left(1-\beta_{1}\right) s\left(\epsilon_{1 c G}-\epsilon_{1 c G}^{\min }\right)}
$$

The critical value $\sigma_{F}$ solves $1+T\left(\sigma_{F}\right)+D\left(\sigma_{F}\right)=0$ :

$$
\sigma_{F}=\frac{\theta(1-s)\left(\bar{\epsilon}_{l G}-1\right)\left(1-\beta_{1}-2 \epsilon_{1 c c}\right)+2 s \bar{\epsilon}_{l w}\left[\left(1-\beta_{1}\right) \epsilon_{1 c G}-\left(1+\beta_{1}\right) \epsilon_{1 c c}\right]}{2\left(1-\beta_{1}\right) s\left(\epsilon_{1 c G}^{\max }-\epsilon_{1 c G}\right)}
$$

Critical values $\bar{\epsilon}_{l w}^{F}$ and $\bar{\epsilon}_{l w}^{H}$

The critical value $\bar{\epsilon}_{l w}^{F}$ solves $S=-1$ :

$$
\bar{\epsilon}_{l w}^{F}=\frac{\theta\left(1-(1-s) \bar{\epsilon}_{l G}\right)\left(1-\beta_{1}-2 \epsilon_{1 c c}\right)}{2 s\left(1-\beta_{1}\right) \epsilon_{1 c G}}
$$

The critical value $\bar{\epsilon}_{l w}^{H}$ solves $\sigma_{H}=\sigma_{F}$ or, equivalently, $S=\frac{1-D(+\infty)}{-2-T(+\infty)}$ :

$$
\bar{\epsilon}_{l w}^{H}=\frac{\theta(1-s)\left(\bar{\epsilon}_{l G}-1\right)\left[2 \epsilon_{1 c c}\left(\epsilon_{1 c G}^{\max }-\epsilon_{1 c G}^{\min }\right)-\left(1-\beta_{1}\right)\left(\epsilon_{1 c G}-\epsilon_{1 c G}^{\min }\right)\right]}{2 s\left[\left(\epsilon_{1 c G}-\epsilon_{1 c G}^{\min }\right)\left(1-\beta_{1}-\left(1+\beta_{1}\right) \epsilon_{1 c c}\right)+\left(\epsilon_{1 c G}-1\right)\left(1-\beta_{1}\right)\left(\epsilon_{1 c G}^{\max }-\epsilon_{1 c G}^{\min }\right)\right]}
$$

Note that when $\bar{\epsilon}_{l w}=0, S=-1$ for $\epsilon_{1 c c}^{F}=\frac{1-\beta_{1}}{2}$ and $\sigma_{H}=\sigma_{F}$ for $\epsilon_{1 c c}^{H}=$ $\frac{\left(1-\beta_{1}\right)^{2}}{8 \beta_{1}}\left[\sqrt{1+\frac{16 \epsilon_{1 c G} s \beta_{1}}{\left(1-\beta_{1}\right)^{2}(1-(1-s) \bar{\epsilon} l G)}}-1\right]$. 


\section{References}

[1] Becker, R. A. (1980), On the Long-Run Steady State in a Simple Dynamic Model of Equilibrium with Heterogeneous Households, Quarterly Journal of Economics 95, 375-382.

[2] Becker, R. A., Bosi, S., Le Van, C. and T. Seegmuller (2012), On the Existence, Efficiency and Bubbles of Ramsey Equilibrium with Borrowing Constraints, AMSE Working Paper 2012-31.

[3] Becker, R. A., Boyd III, J. H. and C. Foias (1991), The Existence of Ramsey Equilibrium, Econometrica 59, 441-460.

[4] Becker, R. A. and C. Foias (1987), A Characterization of Ramsey Equilibrium, Journal of Economic Theory 41, 173-184.

[5] Becker, R. A. and C. Foias (1994), The Local Bifurcation of Ramsey Equilibrium, Economic Theory 4, 719-744.

[6] Blundell, R., and T. MaCurdy (1999), Labour Supply: a Review of Alternative Approaches, in Handbook of Labor Economics, Ashenfelter, O. and D. Card (eds.) North-Holland, Amsterdam, 1559-1695.

[7] Bosi, S. and T. Seegmuller (2010a), On the Ramsey Equilibrium with Heterogeneous Consumers and Endogenous Labor Supply, Journal of Mathematical Economics 46, 475-492. 
[8] Bosi, S. and T. Seegmuller (2010b), On the Role of Progressive Taxation in a Ramsey Model with Heterogeneous Households, Journal of Mathematical Economics 46, 977996.

[9] Cazzavillan, G. (1996), Public Spending, Endogenous Growth, and Endogenous Fluctuations, Journal of Economic Theory 71, 394-415.

[10] Duffy, J., and C. Papageorgiou (2000), A Cross-Country Empirical Investigation of the Aggregate Production Function Specification, Journal of Economic Growth 5, $87-120$.

[11] Dufourt, F., T. Lloyd-Braga and L. Modesto (2008), Indeterminacy, Bifurcations and Unemployment Fluctuations, Macroeconomic Dynamics 12, 75-89.

[12] Dufourt, F., T. Lloyd-Braga and L. Modesto (2011), Sunspot Equilibria and the Expectation-Driven Phillips Curve, Working Paper, Université de Strasbourg, revised version.

[13] Grandmont, J.-M. (2008), Negishi-Solow Efficiency Wages, Unemployment Insurance and Dynamic Deterministic Indeterminacy, International Journal of Economic Theory 4, 247-272.

[14] Grandmont, J.-M., P. Pintus and R. de Vilder (1998), Capital-labour Substitution and Competitive Nonlinear Endogenous Business Cycles, Journal of Economic Theory $\mathbf{8 0 , 1 4 - 5 9 .}$

[15] Gruber, J. (2006) A Tax-Based Estimates of the Elasticity of Intertemporal Substitution, NBER Working Paper 11945. 
[16] Guo, J.-T. and S.G. Harrison (2008), Useful Government Spending and Macroeconomic (In)Stability under Balanced-Budget Rules, Journal of Public Economic Theory 10, 383-397.

[17] Kapour, M. and S. Ravi (2010), Elasticity of Intertemporal Substitution in Consumption: an Empirical Evidence from a Natural Experiment, Working Paper 21, $191-232$.

[18] León-Ledesma, M., P. McAdam and A. Willman (2010), Identifying the Elasticity of Substitution with Biased Technical Change, American Economic Review 100, $1330-1357$.

[19] Le Van, C., M.H. Nguyen and Y. Vailakis (2007), Equilibrium Dynamics in an Aggregative Model of Capital Accumulation with Heterogeneous Agents and Elastic Labor, Journal of Mathematical Economics 43, 287-317.

[20] Le Van, C. and Y. Vailakis (2003), Existence of a Competitive Equilibrium in a One Sector Growth Model with Heterogeneous Agents and Irreversible Investment, Economic Theory 22, 743-771.

[21] Lloyd-Braga, M., L. Modesto and T. Seegmuller (2008), Tax Rate Variability and Public Spending as Sources of Indeterminacy, Journal of Public Economic Theory 10, 399-421.

[22] Mankiw, N.G. (2000), The Savers-Spenders Theory of Fiscal Policy, American Economic Review 90, 120-125. 
[23] Michel, P. and P. Pestieau (1999), Fiscal Policy when Individuals Differ with Regard to Altruism and Labor Supply, Journal of Public Economic Theory 1, 187-203.

[24] Mulligan, C. (2002), Capital Interest and Aggregate Intertemporal Substitution, NBER Working Paper 9373.

[25] Ni, S. (1995), An Empirical Analysis on the Substitutability Between Private Consumption and Government Purchases, Journal of Monetary Economics, 36, 593-605.

[26] Ramsey, F. P. (1928), A Mathematical Theory of Saving, Economic Journal 38, 543-559.

[27] Rogerson, R. and J. Wallenius (2009), Micro and Macro Elasticities in a Life Cycle Model with Taxes, Journal of Economic Theory 144, 2277-2292.

[28] Sorger, G. (2002), On the the Long-Run Distribution of Capital in the Ramsey Model, Journal of Economic Theory 105, 226-243.

[29] Vissing-Jorgensen, A. (2002), Limited Asset Market Participation and the Elasticity of Intertemporal Substitution, Journal of Political Economy 110, 825-853.

[30] Vissing-Jorgensen, A. and O. Attanasio (2003), Stock-Market Participation, Intertemporal Substitution and Risk Aversion, American Economic Review Papers and Proceedings 93, 383-391.

[31] Woodford, M. (1986), Stationary Sunspot Equilibria in a Finance Constrained Economy, Journal of Economic Theory 40, 128-137.

[32] Zhang, J. (2000), Public Services, Increasing Returns, and Equilibrium Dynamics, Journal of Economic Dynamics and Control 24, 227-246. 